\title{
Searching for Light in the Darkness of Winter
}

\author{
During the six-month-long winter night of the South Pole, Nathan Precup \\ oversees operations of the BICEP Array-a radio telescope searching for \\ swirling patterns in the Universe's first light.
}

\author{
By Matteo Rini
}

$\square$ or Nathan Precup, a native of the Pacific

Northwest, carrying out a scientific project in the extreme environment of the South Pole is a personal test of endurance. Along with a small team of co-workers, Precup, a researcher at the University of Minnesota, headed to the South Pole Station in November of last year-the beginning of the austral summer. There, he and the team assembled the Background Imaging of Cosmic Extragalactic Polarization (BICEP) Array. This telescope is the latest version of five generations of instruments aiming to spot patterns in the polarization of the cosmic microwave background that could provide the smoking gun of cosmological inflation. In February, the rest of the team headed home on the last plane out of the South Pole before the nine-month winter season. But Precup

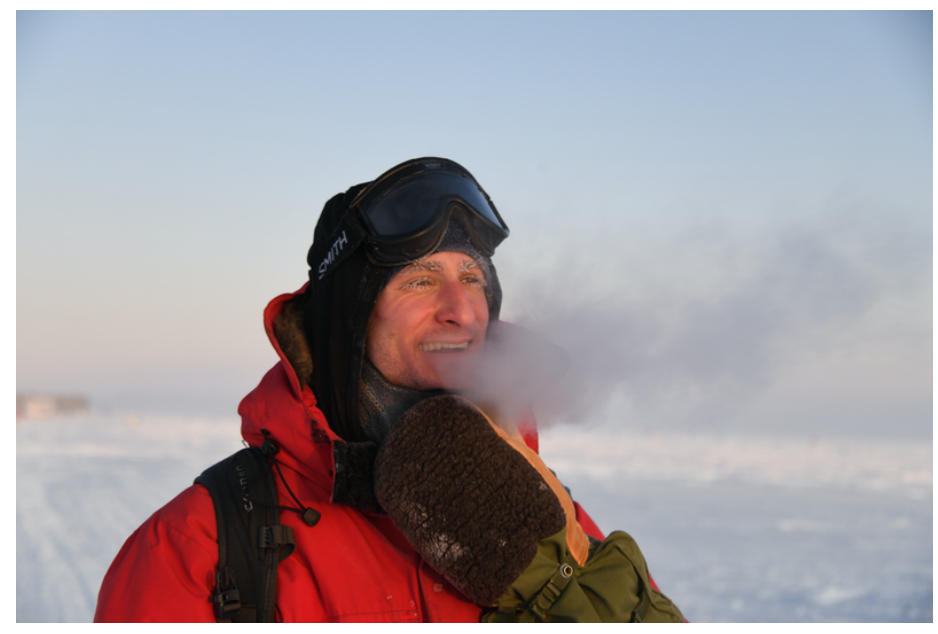

Credit: Y. Makino/IceCube stayed behind to oversee the telescope's operation. Speaking with Physics during one of the precious few-hour windows in which the station gets satellite coverage, Precup described the joys and the challenges of "overwintering" in such an extreme environment.

All interviews are edited for brevity and clarity.

\section{What lured you to the South Pole?}

I wanted to explore my comfort zone and see if I could step away from all the things that I'm used to having-green landscapes, places to hike, the ocean-and still be content and happy. Could I deal with the cold and isolation? I was also really excited about the science and about the idea of working on a telescope that probes the faint light coming from the birth of the Universe.

\section{What did you do before you decided to go on assignment to the South Pole?}

I am an aerospace engineer and I previously worked on wind tunnels and flight tests. My experience with data acquisition systems and software, and my aptness at fixing hardware, is probably why I was chosen as a jack-of-all-trades who can perform the tasks needed to keep the BICEP Array running.

\section{What kind of tasks?}

Together with the rest of the team, I spent the polar summer working like crazy to install a new telescope mount as well as the first of the four receivers that will make up the BICEP Array. Since the others left, my jobs are to ensure that this complicated instrument continues to operate smoothly and to send the data it collects to the researchers at the University of 


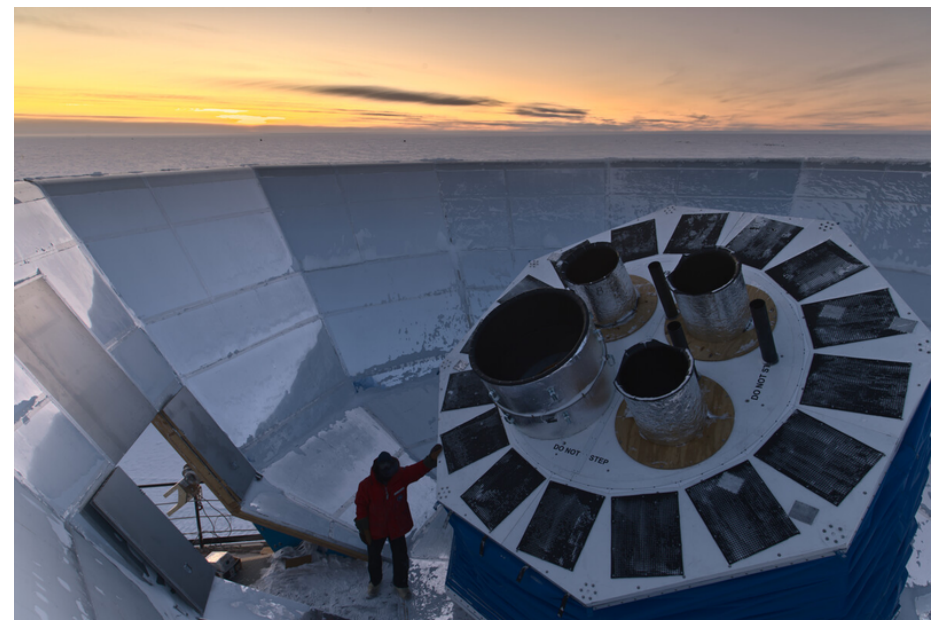

Precup standing beside the first receiver of the BICEP Array.

Credit: N. Precup

Minnesota and at Harvard University. I've had to do a lot of programming to solve some issues with the software that's controlling the telescope. And I've had to solve some mechanical issues with the rotary joints that allow the telescope to rotate and follow the same patch of sky without the need to disconnect the "umbilical cords" that transport data, power, and cryogenic gases.

\section{Which tasks do you like most and which would you prefer not to have to do?}

I really enjoy digging into the software that controls all the different elements of the instrument-the moving parts that control telescope pointing, the data transfer system, and the cryogenic cooling equipment. It's a complex choreography that must be precisely synchronized. My least favorite task is shoveling snow, which I have to do when snow accumulates in the giant reflective bowl that protects the telescope's receivers from radiation from the ground. Luckily that doesn't happen too often-the telescope's location was chosen because of its dryness.

\section{How is winter life at the South Pole station?}

There are 42 winterovers, which include both the scientists and the personnel needed for the station's operation. It's a pretty great community with no "bad actors." We try and keep it fun. The station has a well-equipped gym and a volleyball court where we play games a couple of times a week. We also have

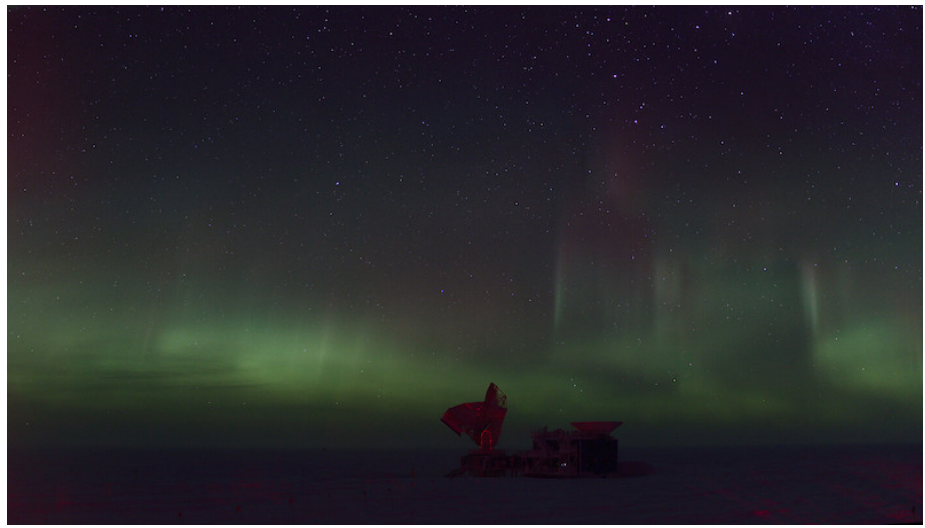

At the South Pole, Precup sees an aurora about every 48 hours. Credit: N. Precup

trivia events, a weekly movie night, and our own version of Iron Chef-a cooking competition inspired by the TV show.

\section{Can you describe the landscape around the station?}

The station stands at an elevation of over 2800 meters, on a flat plateau of snow into which the wind has sculpted tiny white waves. The wavy plane is a beautiful sight in summer, and it reminds me of looking out over the ocean. In winter, the beauty is in the sky. I like to walk outside, with my headlamp turned off, to enjoy the starry sky. And even though I see auroras every other day, the sight of them never fails to impress me, especially when they contain colors other than green, such as red or purple.

\section{What other experiments at the South Pole interest you?}

Climate change is the biggest challenge facing our generation. I am really interested in the climate-related research of the Atmospheric Research Observatory, which takes air samples in the "clean-air sector"-an area that prevailing winds screen from local pollution.

And the IceCube Neutrino Observatory is a fascinating experiment in the "dark sector"-an area kept clear of electromagnetic interferences; the BICEP array also works in this area. One cubic kilometer of ice turned into a detector of ghostly neutrinos-that's super cool. IceCube researchers dream of catching a supernova before anybody else and sending out alerts so that it can be followed through 
multimessenger astronomy.

What's one thing you plan to do when you return home Matteo Rini is the Deputy Editor of Physics. that you can't do here?

Sit outdoors on a park bench staring at the trees. 